\title{
ON THE CLASSIFICATION OF FINITE SIMPLE GROUPS BY THE NUMBER OF INVOLUTIONS
}

\author{
MARCEL HERZOG
}

\begin{abstract}
Simple groups with $k$ involutions, where $k \equiv 1$ (modulo 4), are completely determined.
\end{abstract}

The aim of this note is to prove the following:

THEOREM. Let $G$ be a finite simple group with I involutions and suppose that $I \equiv 1(\bmod 4)$. Then one of the following holds:

(a) $I=1$ and $G$ is cyclic of order 2 ,

(b) $I=105$ and $G \simeq A_{7}$,

(c) $I=165$ and $G \simeq M_{11}$,

(d) $I=q(q+\epsilon) / 2$, and $G \simeq \operatorname{PSL}(2, q)$, where $q=p^{n}>3$ is a power of an odd prime, $\epsilon=1$ or -1 and $q \equiv \epsilon(\bmod 8)$,

(e) $I=q^{2}\left(q^{2}+q+1\right)$ and $G \simeq \operatorname{PSL}(3, q)$, where $q=p^{n}$ is a power of an odd prime and $q \equiv-1(\bmod 4)$,

(f) $I=q^{2}\left(q^{2}-q+1\right)$ and $G \simeq \operatorname{PSU}(3, q)$, where $q=p^{n}$ is a power of an . odd prime and $q \equiv 1(\bmod 4)$.

Proof. By [4], a Sylow 2-subgroup of $G$ is cyclic, generalized quaternion, dihedral of order $\geqslant 8$ or quasi-dihedral. In the cyclic case we get (a). A generalized quaternion Sylow 2-subgroup is impossible by [2] and in the dihedral or quasi-dihedral cases we get (b)-(f) by [3] and [1].

It is easy to check the following:

COROLlaRY. Each of the above mentioned simple groups is characterized by the number of its involutions. In particular, $M_{11}$ is the unique simple group with 165 involutions and $A_{7}$ is the unique simple group with 105 involutions.

Added In Proof. The groups $A_{8}$ and $\operatorname{PSL}(3,4)$ are of the same order and each has 315 involutions. Conjecture: if two simple groups have the same number of involutions, then they are of the same order.

\section{BIBLIOGRAPHY}

1. J. L. Alperin, R. Brauer and D. Gorenstein, Finite groups with quasi-dihedral and wreathed Sylow 2-subgroups, Trans. Amer. Math. Soc. 151 (1970), 1-261.

Received by the editors February 13, 1979.

AMS (MOS) subject classifications (1970). Primary 20D05.

(C) 1979 American Mathematical Society 0002-9939/79/0000-0553/\$01.50 
2. R. Brauer and M. Suzuki, On finite groups of even order whose 2-Sylow group is a quaternion group, Proc. Nat. Acad. Sci. U.S.A. 45 (1959), 1757-1759.

3. D. Gorenstein and J. Walter, The characterization of finite groups with dihedral Sylow 2-subgroups. I, II, III, J. Algebra 2 (1965), 85-151, 218-270, 354-393.

4. M. Herzog, Counting group elements of order p modulo $p^{2}$, Proc. Amer. Math. Soc. 65 (1977), 247-250.

Departigant of Mathiematics, Tel-Aviv University, Tel-Aviv, Israbl 